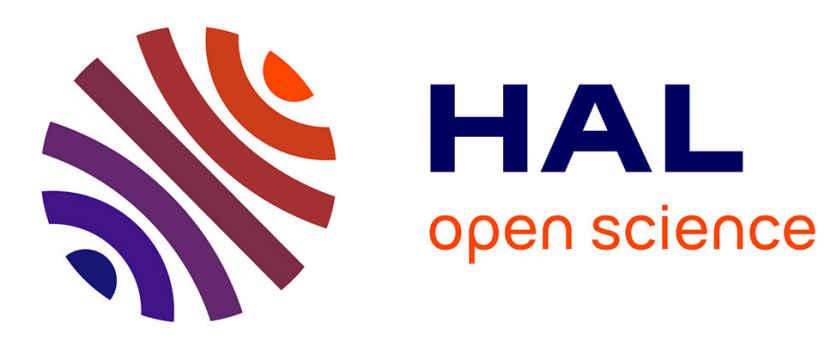

\title{
Sharing beliefs: between agreeing and disagreeing
} Antoine Billot, Alain Chateauneuf, Itzhak Gilboa, Jean-Marc Tallon

\section{To cite this version:}

Antoine Billot, Alain Chateauneuf, Itzhak Gilboa, Jean-Marc Tallon. Sharing beliefs: between agreeing and disagreeing. Econometrica, 2000, pp.685-694. halshs-00174553

\section{HAL Id: halshs-00174553 \\ https://shs.hal.science/halshs-00174553}

Submitted on 25 Sep 2007

HAL is a multi-disciplinary open access archive for the deposit and dissemination of scientific research documents, whether they are published or not. The documents may come from teaching and research institutions in France or abroad, or from public or private research centers.
L'archive ouverte pluridisciplinaire HAL, est destinée au dépôt et à la diffusion de documents scientifiques de niveau recherche, publiés ou non, émanant des établissements d'enseignement et de recherche français ou étrangers, des laboratoires publics ou privés. 


\title{
Sharing Beliefs: B etween A greeing and Disagreeing
}

\author{
By A ntoine Billot, Alain Chateauneuf, Itzhak Gilboa, and J ean-M arc Tallon ${ }^{1}$
}

\section{1. int r oduction}

When is it Pareto optimal for risk averse agents to take bets? Under what conditions do they choose to introduce uncertainty into an otherwise certain economic environment? One obvious case is where they do not share beliefs. As in the classical (theoretical) example of horse lotteries, people who do not agree on probability assessments do ${ }^{-}$nd it mutually bene ${ }^{-}$cial to engage in uncertainty-generating trade.

If the agents involved are Bayesian expected utility maximizers and strictly risk averse, it is not hard to see that disagreement on probabilities is the only way that betting, understood as trade of an uncertain asset, may be Pareto improving when starting from a full insurance allocation. On the other hand, any such disagreement induces betting. Put di ßerently, Pareto optimality dictates either that there be no betting (in case beliefs are common to all agents) or that there be betting (in case of disagreement). This is somewhat puzzling, because there is no lack of allocation-neutral, I sunspot" sources of uncertainty in the world around us. If every disagreement on probabilities of states of the world suggests a Pareto improving trade, one might have expected to see much more betting taking place. 
Rather than believing that people who do not bet necessarily share probabilistic beliefs about anything they do not bet on (or, to be precise, share these beliefs up to some slack allowed by transaction costs), we tend to take the relative rarity of bets as a piece of empirical evidence against the Bayesian model. It seems that often people do not bet because they are uncertainty averse, and they therefore tend to avoid uncertainty that they know little about. It follows that a person's willingness to bet will increase with her subjective con $^{-}$dence in her information and in her likelihood assessments. It is worth emphasizing that Bewley's (1986) motivation for his work on K nightian decision theory was partly this absence of observed widespread betting.

While we do not attempt to argue that the full complexity of betting behavior can be explained by the type of models we study here, ${ }^{2}$ we are led to ask, how much can be explained by these models if we relax some of the more demanding assumptions of the Bayesian model. Speci ${ }^{-}$cally, we consider maxmin expected utility with a non-unique prior (Gilboa and Schmeidler (1989)) that captures Knightian uncertainty (K night (1921)). A ssume that such uncertainty averse agents who are also risk averse, give rise to an economy in which there is no aggregate risk. When does there exist full insurance, i.e., no-bet allocations that are also Pareto optimal? When is it the case that all Pareto optimal allocations are full insurance? Is any betting due to di ßerent beliefs, and, conversely, does a di ßerence in beliefs always trigger some betting?

In the multiple prior model an individual is characterized by a utility 
function and a non-empty, closed and convex set of probability measures. The individual evaluates every act by its expected utility according to each possible probability measure, and chooses an act whose minimal expected utility is the highest. The family of preference relations described by this model strictly contains the relations described by Choquet expected utility with a convex capacity (see Schmeidler (1989)).

Consider now a pair of agents conforming to the multiple prior model. It is an easy extension of the expected utility analysis to show that these agents will not bet against one another if they share at least one prior. Moreover, in a general framework with more than two agents and complex bets possibly involving several of them, it is easy to show, following Dow and Werlang (1992) early intuition, that Pareto optimal allocations are indeed full insurance allocations whenever agents' sets of priors have a non-empty intersection (see, e.g., Tallon (1998), Dana (1998)).

The question of whether the converse to this result holds arises naturally: is commonality of beliefs, in the sense of agents sharing a prior in common, exactly what is needed to explain, within the framework of the multiple prior model, the absence of betting on the many possible sources of \extrinsic" uncertainty? Di ßerently put, is the observation of a Pareto optimal allocation that is immune to sunspots enough to tell us something about the intersection of agents' sets of priors?

It turns out that we can answer this question a \pm rmatively and that the result in the Bayesian model has a conceptually identical counterpart in the multiple prior model. Under the same non-triviality conditions, there 
exists a Pareto optimal full insurance allocation if and only if all Pareto optimal allocations provide full insurance, and this holds if and only if all agents share a prior probability on the states of the world. In other words, commonality of beliefs is the necessary and su \pm cient condition to explain the absence of betting. Whereas in the Bayesian model \sharing a prior" could only mean \having an identical prior," in the multiple prior model this phrase may be read as \having at least one prior in common." With this grammatical convention in place, the result holds verbatim.

Bayesian agents either agree on probability assessments, or disagree enough to bet against each other. By contrast, uncertainty averse agents can be in a Igrey area" between agreeing and disagreeing: they may not agree in the sense of having the same set of possible priors, yet not disagree in the sense of being willing to bet against each other.

Finally, we emphasize another contribution of this note. In showing that commonality of beliefs is the minimal assumption explaining the absence of bets, we prove a separation theorem for $n$ convex sets that might be of interest on its own.

The rest of this paper is organized as follows. Section 2 provides the set up of the model. In section 3 we state the main result and the separation theorem. Proofs are relegated to an appendix.

2. set -up

The economy we consider is a standard two-period pure-exchange economy with uncertainty in the second period, but for agents' preferences. The state space is $S$, and $\S$ a $3 / 4$ al gebra of subsets of $S$, so that $(S ; \S)$ is a measur- 
able state space. There are $\mathrm{n}$ agents indexed by subscript $\mathrm{i}$. We assume (i) that there is only one good, which can be interpreted as income or money; and (ii) that there is no aggregate uncertainty. Trading an uncertain asset is thus interpreted as betting rather than as hedging. Let $\mathrm{B}(\mathrm{S} ; \S)$ be the $\mathrm{Ba}$ nach space of real-valued, bounded and measurable functions on S, endowed with the sup-norm. Let $b a(S ; \S)$ be the space of bounded ' nitely additive measures on $(S ; \S)$ endowed with the weak?-topology. A gent i's consumption $C_{i}$, is a positive element of $B(S ; \S)$, that is, $C_{i}(s)$ is the consumption of agent $\mathrm{i}$ in state $\mathrm{S}$. Denote by w $2 \mathrm{~B}(\mathrm{~S} ; \S)$ the constant-across-states aggregate endowment, and assume that $w>0$. An allocation $C=\left(C_{1} ;:: ; C_{n}\right)$ is feasible if ${ }^{P}{ }_{i=1} C_{i}=w$. An allocation is interior if $C_{i}(s)>0$ for all $i$, for all $\mathrm{s}$.

In the multiple-prior approach, each agent $\mathrm{i}$ is endowed with a utility index $U_{i}: \mathbb{R}_{+} ! \quad \mathbb{R}$ and a set $P_{i}$ of probability distributions over $S . U_{i}$ is de $^{-}$ned up to a positive a \pm ne transformation, and is taken to be di ßerentiable, strictly increasing and strictly concave. $\mathrm{P}_{\mathrm{i}}$ is a convex and closed set of $b a(S ; \S)$. We assume that all priors in $P_{i}$ are $3 / 4$ additive. ${ }^{3}$ Note that $P_{i}$ is compact in the weak?-topology since it is a weak?-closed subset of the set of - nitely-additive probability measures on $\S$, which is compact in the weak?topology (see, e.g., Dunford and Schwarz (1958)). The norm-dual of B (S; § ) which is isometrically isomorphic to $\mathrm{ba}(\mathrm{S} ; \S)$ will be denoted $\mathrm{B}^{\text {? }}(\mathrm{S} ; \S)$.

The overall utility function $V_{i}$ de ${ }^{-}$ned over $B(S ; \S)$ then takes the following form:

$$
V_{i}\left(C_{i}\right)=\min _{1 / \mathbb{P} P_{i}} E_{1 / 4} U_{i}\left(C_{i}\right)
$$


We assume throughout that all priors are mutually absolutely continuous:

$$
8 A 2 \S ; 8 i ; j ; 81 / \mathfrak{4} 2 P_{i} ; 81 / \mathfrak{4} 2 P_{j} ; \quad 1 / \mathfrak{A}(A)=0(\text { ) } 1 / \mathfrak{A}(A)=0
$$

This assumption essentially says that all agents agree on \null events". It is naturally satis ${ }^{-}$ed if, say, the state space is " nite and all measures are strictly positive, or if all measures are absolutely continuous with respect to an underlying measure.

The last de ${ }^{-}$nition we need is that of a full insurance allocation. An allocation $C$ is said to be full insurance if it is constant apart from a set A $2 \S$ that has $1 / \mathfrak{A}(A)=0$ for some (and therefore, by the assumption of mutual absolute continuity, for all) $1 / 42 \mathrm{Pi}_{\mathrm{i}}$ and i. 4

\section{3. the main result}

The following theorem states that the set of Pareto optimal allocations and the set of full insurance allocations are either identical or disjoint. M oreover, they are identical if and only if the agents share at least one prior.

Theor em 1 Under the maintained assumptions, the following assertions are equivalent:

(i) There exists an interior full insurance Pareto optimal allocation.

(ii) Any Pareto optimal allocation is a full insurance allocation.

(iii) Every full insurance allocation is Pareto optimal.

(iv) ${ }_{i=1}^{\top} P_{i} G$;

The intuition for the proof (and the role of some assumptions) is as 
follows. We prove that (iv) ) (ii) ) (iii) ) (i) ) (iv). If there is a common prior (iv), one can use strict concavity to show that a risk bearing allocation is Pareto dominated by the full insurance allocation that equals its expectation at every state, proving (ii). ${ }^{5}$ This step uses the mutual absolute continuity assumption, as well as the assumption that the probability measures we deal with are 3/4additive (rather than only " nitely additive). Observe that with - nitely additive measures the implication (iv) ) (ii) does not hold, even in a Bayesian set-up. This is so because the integral of a function with respect to a " nitely additive measure may be strictly smaller than each of the values the function assumes. Therefore individuals who hold assets that they view as uncertain may not bene ${ }^{-} t$ from smoothing them across states. If every Pareto improving allocation provides full insurance (ii), the converse (iii) also holds, since no two full insurance allocations can be Pareto ranked, ${ }^{6}$ and it follows trivially that there is at least one such allocation (i). Finally, the crucial step and the main contribution of the theorem is that the existence of a full insurance Pareto optimal allocation (i) implies that there is a common prior (iv). This step does not require concavity of the utility index: ${ }^{7}$ In proving this last part we make use of the following theorem, which generalizes the standard separating hyperplane theorem, and may be of interest on its own. In the appendix we also comment on the geometric interpretation of this result which may be viewed as a separation theorem among $\mathrm{n}$ convex sets. 
Theor em 2 Let $X$ be a locally convex linear topological space and let $P_{i} \mu X, 1$ i $n$, be convex, non-empty, and compact. Then, the following are equivalent:

(i) ${ }_{i=1}^{\mathrm{n}} \mathrm{P}_{\mathrm{i}}=$;

(ii) There exist I $\mu f 1 ;::: ; n g, I \notin$; and $p 2 \operatorname{co}\left(\left[{ }_{i 2} \mid P_{i}\right)\right.$ and for each i $2 \mathrm{I}$, there exists a continuous linear functional $h_{i}: X ! I R$ such that:

(a) $8 \mathrm{i} 2 \mathrm{I}, \mathrm{h}_{\mathrm{i}}\left(\mathrm{q}_{\mathrm{i}} \mathrm{p}\right)>0$ for all $\mathrm{q} 2 \mathrm{P}_{\mathrm{i}}$

(b) ${ }_{i 21}^{\mathrm{P}} \mathrm{h}_{\mathrm{i}}=0$

A n immediate corollary of Theorem 2 is that, under the same assumption, if ${ }_{i=1}^{\top} P_{i}=$; , there exist continuous linear functionals $h_{i}, i=1 ;:: ; ; n$ and a point $p$ such that $\left(a^{\prime}\right) h_{i}\left(q_{i} p\right), 0$ for all $q 2 P_{i}$, for all $i,\left(b^{\prime}\right)$ $P_{i=1}^{n} h_{i}=0$, and $\left(c^{\prime}\right)$ there exist $i, i^{0}$ such that the inequality in $\left(a^{\prime}\right)$ is strict.

It is worthy of note that a similar result, developed independently and with a rather di Rerent motivation, is to be found in Samet (1998), for subsets of a ${ }^{-}$nite dimensional simplex. Samet's result is weaker in the sense that it guarantees the existence of linear functionals as in our case, but does not guarantee that the separating hyperplanes will intersect at one point pin the convex hull of the sets, and therefore does not yield itself to a straightforward geometric interpretation. Further, Samet's result can be easily derived from the corollary above specialized to subsets of the simplex. It does not appear that Samet's argument could easily be amended to get ours.

Theorem 1 has two immediate corollaries. F irst, in the Choquet expected 
utility model with convex capacities, non-empty core intersection is equivalent to some, or all, Pareto optimal allocations being full insurance. Second, in the expected utility case, where the sets of priors are reduced to one point, some, or all, Pareto optimal allocations are full insurance allocations if and only if agents have the same beliefs (i.e., the same prior).

Note that even though we cast the argument in the multiple prior model, it should be clear from the proof that a similar result holds for Bewley (1986) approach. In B ewley's approach, agents are also endowed with a set of priors and move away from a (exogenously de ${ }^{-}$ned) status quo situation only if the new situation is better than the status quo for all the probability distributions in their set of priors. While Bewley characterizes a partial order over acts, a proposed bet will be preferred to a certain status quo if and only if this preference holds in the multiple prior model of Gilboa and Schmeidler. 8

Our analysis is conducted for an economy with one good. However, the only use we make of this assumption is in arguing that all full insurance allocations are Pareto optimal. Indeed, one can generalize our results to an economy with $\mathrm{m}$ goods, with the slight modi ${ }^{-}$cation that full insurance allocations that are considered for optimality be assumed Pareto optimal in each state.

CERA S-E NPC and Universit Paris II, 92, rue d'A ssas, 75006 P aris, France. E-mail: billot@u-paris2.fr 
CERM SEM , Universit Paris I, 106-112, bld de l'H ôpital, 75647 P aris Cedex 13, France. E-mail: chateaun@univ-paris1.fr

and

Eitan Berglas School of Economics and Recanati School of Business, TelA viv University, Tel A viv 69978, Israel and Dept of E conomics, Boston University, 270 B ay State Rd, B oston, M A, 02215, U.S.A . E-mail: gilboa@econ.tau.ac.il and CNRS\{E URE Qua, 106-112, bld de l'Hopital, 75647 Paris Cedex 13, France. E-mail: jmtallon@univ-paris1.fr 
appendix

Proof of Theor em 1:

We ${ }^{-}$rst prove (iv) ) (ii) Assume to the contrary that there exists an agent, say, agent 1 , such that for every $1 / 42 \quad P_{1}$ and every $c 2 \quad R_{+}$, $1 / 4\left(f s j C_{1}(s)<c g\right)+1 / 4\left(f s j C_{1}(s) g>c g\right)>0$.

Let $1 / 42 \backslash_{i} P_{i}$ and de ${ }^{-}$ne $C_{i}=E_{1 / 4} C_{i}$ for all $i$. A busing notation, let $C_{i}$ also denote the constant allocation giving $\mathcal{C}_{i}$ to agent $i$ in all states. $C=\left(C_{i}\right)_{i}$ is a feasible allocation since ${ }^{P}{ }_{i} C_{i}={ }_{i}^{P} E_{1 / 4} C_{i}=E_{1 / 4}\left({ }_{i}{ }_{i} C_{i}\right)=E_{1 / 4} w l_{S}=w$. Now,

$$
V_{i}\left(C_{i}\right)=\min _{2 P_{i}} E_{1} U_{i}\left(C_{i}\right) \quad E_{1 / 4} U_{i}\left(C_{i}\right)
$$

Furthermore,

$$
E_{1 / 4} U_{i}\left(C_{i}\right) \quad U_{i}\left(E_{1 / 4}\left(C_{i}\right)\right)=U_{i}\left(C_{i}\right)=V_{i}\left(C_{i}\right)
$$

for all $i$ since $U_{i}$ is concave.

Since $1 / 4$ belongs to $P_{i}$, one gets that

$$
1 / 4^{i} f s j C_{1}(s)<C_{1} g^{\phi}+1 / 4^{i} f s j C_{1}(s)>C_{1} g^{\phi}>0
$$

Furthermore, $1 / 4{ }^{i}$ fsj $C_{1}(s)<C_{1} g^{\phi}=0$ is impossible, for then, $1 / 4$ fsj $C_{1}(s)>C_{1} g^{\phi}>$ 0 , implying by $3 / 4$ additivity of $1 / 4$ that $E_{1 / 4}\left(C_{1}\right)>C_{1}$, a contradiction. Hence, ${ }_{1 / 4}^{i} \mathrm{fsj}_{1}(\mathrm{~s})<\mathrm{C}_{1} \mathrm{~g}^{\phi}>0$ and, similarly, ${ }^{1}{ }_{4}^{\mathrm{i}} \mathrm{fsj}_{\mathrm{C}}(\mathrm{s})>\mathrm{C}_{1} \mathrm{~g}^{\phi}>0$.

It follows that $V_{1}\left(C_{1}\right)<V_{1}\left(C_{1}\right)$ since $U_{1}$ is strictly concave. Therefore, the allocation $C$ Pareto dominates $C$, a contradiction.

To see that (ii) implies (iii), let $\mathrm{C}$ be a full insurance allocation. A ssume, contrary to (iii), that it is not Pareto optimal, and is dominated by another 
allocation $C^{0}$. By the same argument as above, $C^{0}$ is at least as desirable as $\mathrm{C}^{0}$ for every agent. By transitivity of Pareto domination, $\mathrm{C}^{0}$ Pareto dominates $\mathrm{C}$. But this is a contradiction since both provide full insurance and there is only one good in the economy.

That (iii) implies (i) is obvious, and it remains to prove that (i) implies (iv). Suppose to the contrary that $\backslash{ }_{i} P_{i}=$; , and let $C$ be an interior Pareto optimal allocation that is a full-insurance allocation $\left(C_{i}\right.$ is constant for all $i$ apart on a set of measure zero, the latter notion being de- ned unambiguously given our absolute mutual continuity assumption). By Theorem 2 (where $X$ is $B^{?}(S ; \S)$ endowed with the weak? -topology), since $\backslash_{i} P_{i}=$; , there exists a non-empty set $\mathrm{I}$, a point $\mathrm{p}$ and functionals $\mathrm{h}_{\mathrm{i}} 2 \mathrm{~B}$ ? $(\mathrm{S} ; \S)$, i 2 I such that:

(a) $8 \mathrm{i} 2 \mathrm{I}, \mathrm{h}_{\mathrm{i}}\left(\mathrm{q}_{\mathrm{i}} \mathrm{p}\right)>0$ for all $\mathrm{q} 2 \mathrm{P}_{\mathrm{i}}$

(b) ${ }_{i 21}^{\mathrm{P}} \mathrm{h}_{\mathrm{i}}=0$

Recall that (see e.g. Kelley and Namioka (1963), p.155) every weak?continuous linear functional on the conjugate space of a linear topological space $E$ is the evaluation at some point of $E$. Hence, for all i $2 \mathrm{I}$, there exists $D_{i} 2 B(S ; \S)$ such that $h_{i}(p)=p\left(D_{i}\right)$, for all $p 2 B$ ? $(S ; \S)$.

Construct the allocation $\dot{b}_{i=1 ; \ldots ;, n}$ as follows:

$$
\begin{aligned}
& \dot{b}_{i}=C_{i} \quad i z \mid \\
& \dot{b}_{i}=C_{i}+"\left[D_{i} i p\left(D_{i}\right) 1_{s}\right] \text { i } 2 \text { I }
\end{aligned}
$$

with " $>0$ small enough so that $£$ is an allocation.

We ${ }^{-}$rst check that this allocation is feasible:

$$
"{ }^{x} D_{i} i_{i 21}{ }^{x} p\left(D_{i}\right) 1_{S}={ }^{\#}{ }_{i 21}^{x} D_{i}{ }^{x}{ }_{i 21}^{x} h_{i}(p){ }^{\#}
$$




$$
={ }^{\mathrm{X} 21} \mathrm{D}_{\mathrm{i}} \text { since }{ }_{\mathrm{i} 21}^{\mathrm{X}} \mathrm{h}_{\mathrm{i}}=0
$$

Now, $D_{i}$ is such that $h_{i}(q)=q\left(D_{i}\right)$ for all $q 2 B ?(S ; \S)$ and hence $\left.q^{P} i 2, D_{i}\right)=0$ for all $q 2 B^{?}(S ; \S)$.

To concludethat ${ }^{P}{ }_{i 21} D_{i}=0$, suppose there exists s such that ${ }^{P}{ }_{i 21} D_{i}(s)=$ $a, a \in 0$. The event $f s^{P}{ }_{i 21} D_{i}(s)=$ ag is measurable because the $D_{i}$ are measurable. Now, let $q$ be the continuous linear functional in $B$ ? $(S ; \S)$ corresponding to the additive probability in $\mathrm{ba}(\mathrm{S} ; \S)$ with the mass 1 on that event. Then $\left.\mathrm{q}^{\mathrm{P}}{ }_{\mathrm{i} 21} \mathrm{D}_{\mathrm{i}}\right)=0$ implies $\mathrm{a}=0$, a contradiction. Hence, $P_{i 21} D_{i}=0$.

Now, for i 2 I, one has:

$$
\begin{aligned}
& V_{i}\left(\dot{\phi}_{i}\right)=E_{q^{\prime \prime}} U_{i}\left(C_{i}+"\left[D_{i} i p\left(D_{i}\right) 1_{S}\right]\right) \text { for some } \hat{q} " 2 P_{i} \\
& =V_{i}\left(C_{i}\right)+" U_{i}\left(C_{i}\right)\left[\hat{q}\left(D_{i}\right) i p\left(D_{i}\right)\right]+o(") \\
& \left.=V_{i}\left(C_{i}\right)+" U_{i}^{q}\left(C_{i}\right)\left[h_{i}\left(q^{\prime \prime} i \quad p\right)\right]+o^{\prime \prime}\right) \\
& \text {, } V_{i}\left(C_{i}\right)+" U_{i}^{q}\left(C_{i}\right)\left[\inf _{q 2 P_{i}} h_{i}\left(q_{i} p\right)+\mathbb{Q}(")\right]
\end{aligned}
$$

where $\mathbb{(}(")=\frac{\mathrm{O}(")}{" !} ! 0$ as "! 0 .

Since inf ${ }_{\mathrm{q} 2 \mathrm{P}_{\mathrm{i}}} \mathrm{h}_{\mathrm{i}}\left(\mathrm{q}_{\mathrm{i}} \mathrm{p}\right)>0$ by continuity of $h_{i}$ and compactness of $\mathrm{P}_{\mathrm{i}}$, and ®") ! 0, there exists " small enough so that the term in bracket is strictly positive.

Hence, $V_{i}{ }^{3} e_{i}>V_{i}\left(C_{i}\right)$ for i 2 l, and we found a Pareto dominating allocation $\mathrm{b}_{i=1 ; \ldots ; n}$, a contradiction.

Q:E :D:

Proof of Theor em 2: We start with the following lemma:

Lemma: Let $X$ be a locally convex linear topological space and let $P_{i} \mu X$, 1 i n be convex, non-empty, and compact. Assume that $\backslash_{\mathrm{i}}{ }_{\mathrm{n}} \mathrm{P}_{\mathrm{i}}=$; 
but that for all $\backslash n, \backslash_{i} \cdot P_{i} \in ;$. Then, there exist $p 2 \operatorname{co}\left(\left[{ }_{i=1}^{n} P_{i}\right)\right.$ and $a$ continuous linear functional $h_{i}: X ! I R$ for each $i$ n such that:

(a) $8 \mathrm{i} \quad n, h_{i}\left(q_{i} \quad p\right)>08 q 2 P_{i}$

(b) ${ }^{\mathrm{P}}{ }_{\mathrm{n}} \mathrm{h}_{\mathrm{i}}=0$

The geometric interpretation of this lemma is as follows. A ssume that $n$ convex and compact sets have an empty intersection, but that every subset of them has a non-empty intersection. Then, we can ${ }^{-}$nd a point $\mathrm{p}$ which is not included in any set, but which is \in the middle" in the following sense: one can ${ }^{-}$nd, for each set $\mathrm{P}_{\mathrm{i}}$, a hyperplane $\mathrm{h}_{\mathrm{i}}$ that passes through $\mathrm{p}$ which is in the convex hull of the union of the $\mathrm{P}_{\mathrm{i}}$ and leaves the entire $\mathrm{P}_{\mathrm{i}}$ on one side, such that the normals of these hyperplanes, multiplied by appropriate positive constants, add up to zero. In the case $\mathrm{n}=2$, our lemma reduces to a standard separation theorem between two disjoint sets. For $n>2$, the lemma may be considered as an n-way separation among $\mathrm{n}$ convex sets. See - gure 1 for an illustration of the case $n=3$.

\section{Please insert Figure 1 here}

Proof of the I emma: The proof is by induction on $n$. For $n=2$, we have $P_{1} \backslash P_{2}=$; and we use a standard separation theorem (cf Kelley and Namioka (1963), p.119, theorem on strong separation) to conclude that there is a continuous linear functional $h: X ! \mathbb{R}$ and a number ${ }^{-} 2 \mathbb{R}$ such that $h(q)>^{-}$for $q 2 P_{1}$ and $h(q)<{ }^{-}$for $q 2 P_{2}$. Choose $p$ such that $h(p)={ }^{-}$, and set $h_{1}=h$ and $h_{2}=i h$. By linearity of $h$ it is possible to choose $\mathrm{p} 2 \operatorname{co}\left(\mathrm{P}_{1}\left[\mathrm{P}_{2}\right)\right.$. 
A ssume that the lemma holds for every $n^{0}<n$. Let there be given $\left(P_{i}\right)_{i=1}^{n}$. Set $A=\backslash_{i<n} P_{i}$ and $B=P_{n}$. Observe that $A$ and $B$ are convex, non-empty, and compact. Furthermore, they are disjoint since $\backslash_{i} P_{i}=$; . A pply the same separation theorem to conclude that there exist a continuous linear $R_{n}: X ! \quad \mathbb{R}$ and ${ }^{-} 2 \mathbb{R}$ such that

$$
R_{n}(q)>^{-} 8 q 2 B \text { and } R_{n}(q)<^{-} 8 q 2 A
$$

Choose $q_{0} 2 \times$ such that $R_{n}\left(q_{b}\right)={ }^{-}$. We shift the origin to $q_{0}$. Speci cally, de $^{-}$ne for each $i \quad n, \Phi_{i}=f p_{i}$ oj j p $2 P_{i} g=P_{i} i \quad q_{0}$. Naturally, $\oplus_{i}{ }_{i=1}^{n}$ and their intersections inherit all relevant properties of $\left(\mathrm{P}_{\mathrm{i}}\right)_{\mathrm{i}}$. Denote $\mathrm{B}=$ $B ; \quad q_{0}=\Phi_{n}$ and $\phi=A ; \quad q_{0}=\backslash_{i<n} \Phi_{i}$ and observe that $R_{n}(q)>08 q 2 B$ and $R_{n}(q)<08 q 2$ A. Consider $X^{0}=f q 2 \times j R_{n}(q)=0 g . X^{0}$ is a locally convex linear topological subspace of $X$. Focusing on this subspace, de ${ }^{-}$ne $\Phi_{i}^{0}=\Phi_{i} \backslash X^{0}$ for $\mathrm{i}<\mathrm{n}$. Obviously, $\Phi_{\mathrm{i}}^{0}$ is convex and compact for every $\mathrm{i}<\mathrm{n}$. We argue that it is also non-empty. Indeed, $\Phi_{\mathrm{i}}$ contains $\mathbb{R}$. On the other hand, $\mathbb{\Phi}_{i}$ has a non-empty intersection with $B=\mathbb{D}_{n}$. By convexity of

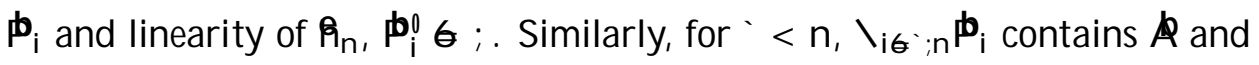
intersects $B$ and we therefore get

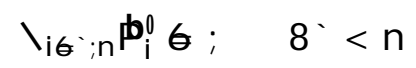

However, $X^{0}$ is an hyperplane separating $B^{\circ}$ from $\mathcal{A}$. Hence $\backslash_{i<n} \mathbb{P}_{i}^{0}=;$. It follows that ${ }^{3} \mathrm{p}_{\mathrm{i}}^{0}{ }_{i<n}$ on $\mathrm{X}^{0}$ satisfy the conditions, of the lemma for $\mathrm{n}^{0}=$ $n_{i}$ 1. Therefore, there exist a point $\mathbf{p} 2$ co $\left[\prod_{i=1}^{n_{i}}{ }_{1} \phi_{i}\right.$ and continuous linear functionals $h_{i}^{0}: X^{0}$ ! $\mathbb{R}, i<n$, such that $h_{i}^{0}\left(q_{i} p\right)>08 q 2 \Phi_{i}^{0}, i<n$, and ${ }^{P} i<n h_{i}^{0}=0$ on $X^{0}$. Using standard arguments (see Fact 1 below), we 
conclude that, for every $\mathrm{i}<\mathrm{n}, \mathrm{h}_{\mathrm{i}}^{0}$ on $\mathrm{X}^{0}$ can be extended to $\mathrm{h}_{\mathrm{i}}$ on all of $\mathrm{X}$ such that:

$$
h_{i}\left(q_{i} \quad p\right)>0 \quad 8 q 2 p_{i}
$$

$D e^{-}$ne $h={ }^{P}{ }_{i<n} h_{i}$ on $X$. Observe that for every $q 2 X^{0}$,

$$
h(q)={ }_{i<n}^{x} h_{i}(q)={ }_{i<n}^{x} h_{i}^{q}(q)=0
$$

Hence $A_{n}$ and $h$ are continuous linear functionals on $X$ satisfying:

$$
\left.R_{n}(q)=0\right) \quad h(q)=0 \quad 8 q 2 x
$$

By standard arguments (see Fact 2 below), there exists ${ }^{\circledR} 2 \mathbb{R}$ such that $h(q)=\circledast R_{n}(q) 8 q 2 X$.

We wish to show that $®<0$. Consider $q 2 \not R=\backslash_{i<n} \mathbb{P}_{i}$. Since $h_{i}\left(q_{i} \quad p\right)>$ $08 \mathrm{i}<\mathrm{n}$ and $\mathrm{h}(\mathrm{p})=0$, we obtain

$$
h(q)=h\left(q_{i} p\right)={ }_{i<n}^{x} h_{i}\left(q_{i} p\right)>0
$$

On the other hand, $R_{n}(q)<0$ since $q 2 \notin$. It follows that $\AA<0$.

$$
\text { De } e^{-} \text {ne } h_{n}=(i \circledast) R_{n} \text {. Since }(i \circledast)>0, h_{n}(q i \quad p)=h_{n}(q)>0,8 q 2 \phi_{n} \text {. }
$$

To conclude, set $p=p+q_{0}$. Observe that $p 2$ co $\left[{ }_{i=1}^{n_{i}}{ }_{1}^{1} P_{i}\right.$ and hence p 2 co $\left(\left[n_{i=1}^{n} P_{i}\right)\right.$. We claim that $p$ and $\left(h_{i}\right)_{i}$ n satisfy $(a)$ and $(b)$. Indeed, for every $\mathrm{i} n$, and every $q 2 \mathrm{P}_{\mathrm{i}}$ :

$$
h_{i}\left(q_{i} p\right)=h_{i}\left(q_{i} q_{p}\right) i\left(p_{i} q_{p}\right)=h_{i}\left(q_{i} q_{p}\right) i p>0
$$

since $q_{i} q_{0} 2 \oplus_{i}$. Finally, ${ }^{P}{ }_{i} h_{i}=0$ by construction of $h_{n}$. Q:E:D:

The following two facts, which are used in the proof above, are straightforward and/ or well-known. 
Fact 1: Let $X$ be a locally convex linear topological space. Let $h$ be a continuous linear functional and $X^{0}=f p 2 \times j h(p)=0 g$. Assume that $C \mu \mathrm{X}$ is convex and compact, and that $\mathrm{C} \backslash \mathrm{X}^{0} \mathrm{G} ;$. Further assume that $h^{0}: X^{0} ! \mathbb{R}$ is a continuous linear functional such that $h^{9}(p)>0$ $8 p 2 \mathrm{C} \backslash \mathrm{X}^{0}$. Then, $\mathrm{h}^{0}$ can be extended to a continuous linear functional $h: X ! \quad \mathbb{R}$ such that $h(p)>08 p 2 C$.

Proof of Fact 1 : Set $D=f p 2 \times{ }^{0} j h^{q}(p)=0 g$. Observethat $D G$; since the origin is in $\mathrm{D}$. Thus $\mathrm{C}$ and $\mathrm{D}$ are disjoint non-empty closed and convex sets in $\mathrm{X}$, and $\mathrm{C}$ is compact. Let a continuous linear functional $\mathrm{R}: \mathrm{X} ! \mathbb{R}$ and $d 2 \mathbb{R}$ be such that:

$$
R(p)<d \quad 8 p 2 D \text { and } R(p)>d \quad 8 p 2 C
$$

We claim that $R$ has to be constant on D. Indeed, assume that for some $p ; q 2 D, R(p) \in R(q)$. Since $p ; q 2 D$ implies $h(p)=h(q)=0$ and $h^{q}(p)=$ $h q q)=0$, we conclude that $p+\AA(q i$ p) $2 D$ for all $® 2 \mathbb{R}$. Hence $f R(p+\&(q i p)) j \circledast 2 \mathbb{R g}=\mathbb{R}$, a contradiction to the fact that $R(p)<d$ $8 p 2 D$. Thus there is a $c 2 \mathbb{R}$ such that $A(p)=c 8 p 2 D$. Since the origin is in $D$, we obtain $C=0$. It follows that $d>0$ and therefore

$$
R(p)>d>0 \quad 8 p 2 C
$$

We now wish to show that, up to multiplication by a positive constant, $\mathrm{A}$ extends $h^{0}$ on $X$. Restrict attention to $X^{0}$. If $p 2 X^{0}$ satis $^{-}$es $h 9(p)=0$, then p 2 D and we know that $R(p)=0$. By Fact 2 below, there exists $® 2 \mathbb{R}$ such that $R(p)={ }^{2} h(p) 8 p 2 \times 0$. However, on $C \backslash X^{0}$, both $R$ and $h^{0}$ are positive. Therefore ${ }^{\circledR}>0$. Hence $h^{\prime} \frac{1}{\circledR} R$ extends $h^{0}$ on $X$ and is positive 
on all of $C$.

Q:E :D:

Fact 2: Let $X$ be a linear space and let $\AA ; h: X \quad ! \quad \mathbb{R}$ be linear. A ssume that

$$
h(q)=0) \quad h(q)=0 \quad 8 q 2 x
$$

Then there exists ${ }^{\circledR} 2 \mathrm{IR}$ such that $\mathrm{h}(\mathrm{q})={ }^{\circledR} \mathrm{R}(\mathrm{q}) 8 \mathrm{q} 2 \mathrm{X}$

We skip the proof of this Fact and now turn to the proof of Theorem 2:

(i) ) (ii). Assume that $\backslash_{i}{ }_{n} P_{i}=;$. Let I be a minimal (with respect to set inclusion) subset of $f 1 ;:: ;$;ng with the property that $\backslash_{i 21} P_{i}=;$. Since $\backslash \mathrm{i}_{\mathrm{i}=1} \mathrm{P}_{\mathrm{i}}=;$, but $\mathrm{P}_{\mathrm{i}} \sigma$; for every $\mathrm{i}$, such a set I exists and for every such set jl j, 2. Apply the Lemma to I.

(ii) ) (i). A ssume that a point $\mathrm{p} 2 \mathrm{X}$, a set $\mathrm{I} \underline{1 / 2} 1 ;::$ :; ng and functionals $\left(h_{i}\right)_{i 21}$ exist as required, and suppose, contrary to (i), that there exists $q 2 \backslash_{i}{ }_{n} P_{i}$. Then, by $(a),{ }_{i 21} h_{i}\left(q_{i} p\right)>0$, contrary to (b). $Q: E: D:$ 


\section{References}

T. Bewl ey (1986): IK nightian decision theory: Part I," Discussion Paper 807, Cowles Foundation.

| | | \{ (1989): IMarket innovation and entrepreneurship: a Knightian view," Discussion Paper 905, Cowles Foundation.

A. Chat eauneuf, R.A. Dana, and J.-M. Tal I on (1997): IOptimal risksharing rules and equilibria with Choquet expected utility," Cahiers EcoMaths 97-54, Universit Paris I.

R.A. Dana (1998): \Pricing rules when agents have non-additive expected utility and homogeneous expectations," Cahier du Ceremade, Universit Paris IX .

J . Dow and S. Werl ang (1992) IUncertainty aversion, risk aversion, and the optimal choice of portfolio," Econometrica, 60(1), 197\{204.

N. Dunford and J .T. Schwartz (1958): Linear operators. Part I. Interscience, New-York.

I. Gil boa and D. Schmeidl er (1989): \Maxmin expected utility with a non-unique prior," J ournal of Mathematical E conomics, 18, 141\{153.

J. Kel I ey and I. Namioka (1963): Linear topological spaces. Volume of Graduate Text in M athematics, Springer Verlag.

F. Knight (1921): Risk, uncertainty and $\mathrm{pro}^{-} \mathrm{t}$. Houghton Mi $\pm \mathrm{n}$. 
P. Milgrom and N. St okey (1982): IInformation, trade and common knowledge," J ournal of E conomic Theory, 26, $17\{27$.

D. Samet (1998): ICommon priors and separation of convex sets," Games and Economic Behavior, 24, 172\{174.

D. Schmeidl er (1989): \Subjective probability and expected utility without additivity," Econometrica, 57(3), 571\{587.

J.-M. Tal I on (1998): I Do sunspots matter when agents are Choquetexpected-utility maximizers?" J ournal of Economic Dynamics and Control, 22, $357\{368$. 


\section{Footnotes}

1. We thank participants of the Erasmus conference at Tilburg University and two referees for useful comments.

2. In particular, we ignore the social aspects of betting as well as the strategic ones (see, e.g., Milgrom and Stokey (1982)).

3. Note that the axiomatization of Gilboa and Schmeidler (1989) delivers only " nitely additive probability distributions.

4. It is straightforward to check that $C$ is of full-insurance if and only if 8i; $C_{i}$ is constant apart from a set $A_{i} 2 \S$ that has $1 / \not\left(A,\left(A_{i}\right)=0\right.$ for some (and therefore, by assumption of mutual absolute continuity, for all) $1 / 42 P_{i}$.

5. This implication follows the logic of similar results for Choquet expected utility in Chateauneuf, Dana, and Tallon (1998).

6. The fact that (iv) implies (ii) and (iii) also appears in Dana (1998) but in a ${ }^{-}$nite set-up.

7. Dana (1998) shows that if there is a full insurance competitive equilibrium in this economy with " nitely many states, then agents share a prior in common. Her proof, however, uses the concavity of the utility index and relies on the existence of a competitive equilibrium. 
8. Bewley (1989) contains a similar no-trade result for agents whose preferences are given by partial orders as in Bewley (1986). His proof is very similar to Samet's, and his result is weaker than Theorem 2 in the same sense that Samet's is. 
F igure 1:

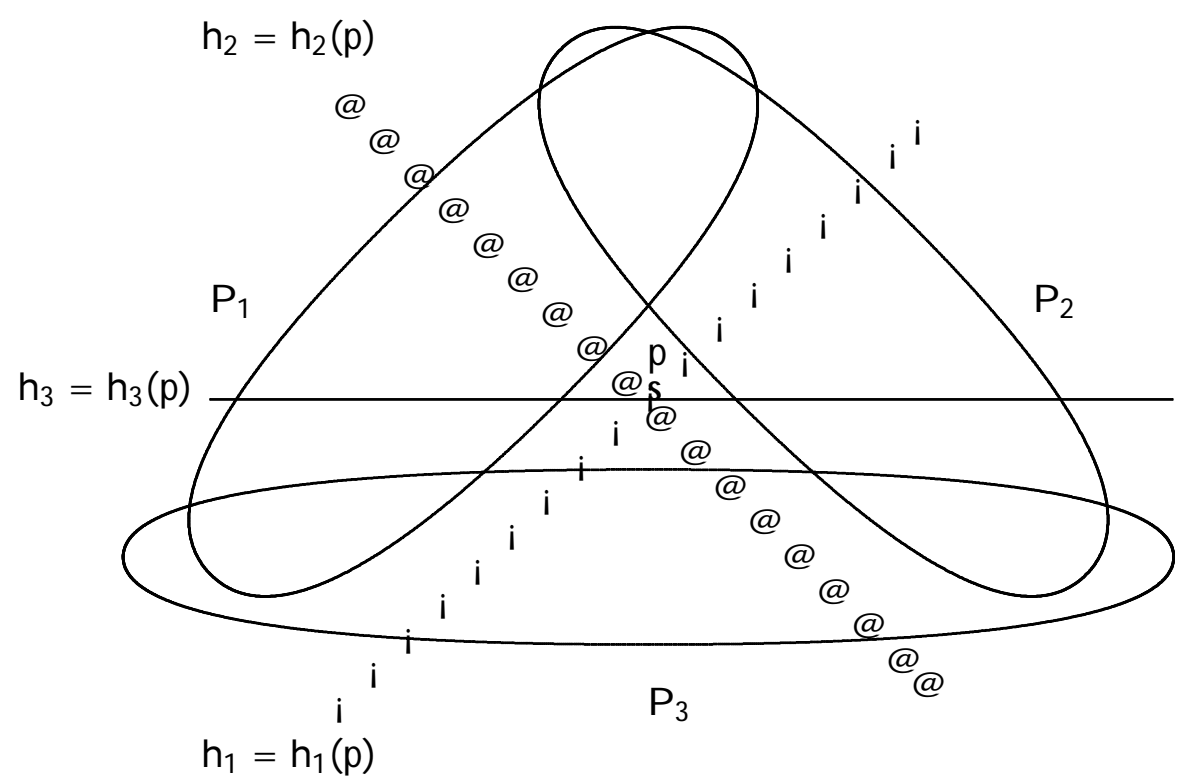

Figur e 1-Separation among three convex sets. 